\title{
ORDER-INVARIANT PRIOR SPECIFICATION IN BAYESIAN FACTOR ANALYSIS
}

\author{
DENNIS LEUNG AND MATHIAS DRTON
}

\begin{abstract}
Using lower triangular loading matrices in Bayesian factor analysis ensures identifiability but may lead to inferences that depend on how the considered variables are ordered. We show how a standard approach to prior specification can be modified to avoid order-dependence.
\end{abstract}

\section{INTRODUCTION}

Let $y$ be an $m$-vector of observed random variables, which for simplicity we take to be centered. Let $f \sim N_{k}\left(0, I_{k}\right)$ be a standard normal $k$-vector of latent factors, with $k \leq m$. The factor analysis model postulates that

$$
y=\beta f+\varepsilon,
$$

where $\beta=\left(\beta_{i j}\right) \in \mathbb{R}^{m \times k}$ is an unknown loading matrix, and $\varepsilon \sim N_{m}(0, \Omega)$ is an $m$-vector of normally distributed error terms that are independent of $f$. The error terms are assumed to be mutually independent with $\Omega=\operatorname{diag}\left(\omega_{1}^{2}, \ldots, \omega_{m}^{2}\right)$ comprising $m$ unknown positive variances that are also known as uniquenesses. This model with an unrestricted $m \times k$ loading matrix $\beta$ is sometimes referred to as exploratory factor analysis - in contrast to confirmatory factor analysis, which refers to situations in which some collection of entries of $\beta$ is modeled as zero.

Integrating out the latent factors $f$ in (1.1), the observed random vector $y$ is seen to follow a centered multivariate normal distribution with covariance matrix

$$
\Sigma=\Omega+\beta \beta^{\prime} .
$$

As discussed in detail in Anderson and Rubin (1956), $\Sigma$ determines the unrestricted loading matrix $\beta$ only up to orthogonal rotation. Indeed, $\beta \beta^{\prime}=\beta Q Q^{\prime} \beta^{\prime}$ for any $k \times k$ orthogonal matrix $Q$. More details on factor analysis can be found, for instance, in Bartholomew et al. (2011), Drton et al. (2007), and Mulaik (2010).

In this paper, we are concerned with Bayesian inference in (exploratory) factor analysis. In Bayesian computation, it is convenient to impose an identifiability constraint on the loading matrix $\beta$. A common choice is to restrict $\beta$ to be lower triangular with nonnegative diagonal entries, that is, $\beta_{i j}=0$ for $1 \leq i<j \leq k$ and $\beta_{i i} \geq 0$ for $1 \leq i \leq k$; see Aguilar and West (2000), Geweke and Zhou (1996), Lopes and West (2004) and Chapter 12 in Congdon (2001). Under these constraints, a full rank matrix $\beta$ is uniquely determined by $\beta \beta^{\prime}$. In the papers just referenced and also the software implementation provided by Martin et al. (2011), a default prior

2000 Mathematics Subject Classification. 62H05.

Key words and phrases. Exploratory factor analysis, latent factor model, permutation invariance. 
on the lower triangular loading matrix has all its non-zero entries independent with

$$
\beta_{i j} \sim \begin{cases}T N\left(0, C_{0}\right) & \text { if } i=j, \\ N\left(0, C_{0}\right) & \text { if } i>j .\end{cases}
$$

Here, $T N\left(0, C_{0}\right)$ denotes a truncated normal distribution on $(0, \infty)$, i.e., the conditional distribution of $X$ given $X>0$ for $X \sim N\left(0, C_{0}\right)$. The variance $C_{0}>0$ is a hyperparameter. The prior distribution for the uniquenesses has $\omega_{1}^{2}, \ldots, \omega_{m}^{2}$ independent of $\beta$ and also mutually independent with Inverse Gamma distribution,

$$
\omega_{i}^{2} \sim \operatorname{IG}\left(\nu / 2, \nu s^{2} / 2\right)
$$

for hyperparameters $\nu, s>0$. Equivalently, $\nu s^{2} / \omega_{i}^{2}$ is chi-square distributed with $\nu$ degrees of freedom; compare Eqn. (26) in Geweke and Zhou (1996).

In the approach that was just described and that will be in the focus of this paper, the prior for the loadings is derived from centered normal distributions with common variance. While this can be restrictive, it is a frequently used default, possibly due to a lack of prior information that warrants non-zero means or unequal variances; see e.g. Section 6 in Ansari et al. (2002). This said, many generalizations have been discussed. For instance, Ghosh and Dunson (2009), Bhattacharya and Dunson (2011), and Conti et al. (2014) present methods to capture sparsity in loading matrices. Other extensions consider $t$-distributed latent factors (Ando, 2009), nonparametric Bayes techniques (Paisley and Carin, 2009) and problems with temporal dependence, see e.g., Nakajima and West (2013), Zhou et al. (2014).

As discussed in Lopes and West (2004, Sect. 6), the prior specification in (1.3) is such that the induced prior on $\beta \beta^{\prime}$ depends on the way the variables and the associated rows of the loading matrix $\beta$ are ordered. Indeed, a priori,

$$
\left(\beta \beta^{\prime}\right)_{i i} / C_{0}=\sum_{j=1}^{k} \beta_{i j}^{2} / C_{0}=\sum_{j=1}^{\min \{i, k\}} \beta_{i j}^{2} / C_{0}
$$

follows a chi-square distribution with degrees of freedom $\min \{i, k\}$. Consequently, the implied prior and also the posterior distribution for the covariance matrix $\Sigma$ from $(1.2)$ is not invariant under permutations of the variables.

In this paper we propose a modification of the prior distribution for $\beta$ that maintains the convenience of computing with an identifiable lower triangular loading matrix all the while making the prior distributions of $\beta \beta^{\prime}$ and $\Sigma$ invariant under reordering of the variables. Our proposal, described in Section 2 merely changes the prior distributions of the diagonal entries $\beta_{i i}$ in $(1.3)$, which will be taken from a slightly more general family than the truncated normal. The details of a Gibbs sampler to draw from the resulting posterior are given in Section 3 . Numerical examples are shown Section 4 . We conclude with a discussion in Section 5 , where we emphasize in particular that the role of lower-triangular loading matrices is a computational one; other ways of mapping the covariance matrix $\Sigma$ to a (unique) loading matrix $\beta$ can be considered when defining a target of inference.

\section{ORDER-INVARIANT PRIOR DISTRIBUTION}

Without any identifiability constraints, the loading matrix $\beta$ takes its values in all of $\mathbb{R}^{m \times k}$. A natural default prior would then be to take all entries $\beta_{i j}, i=$ $1, \ldots, m$ and $j=1, \ldots, k$, to be independent $N\left(0, C_{0}\right)$ random variables; we write $\beta \sim N_{m \times k}\left(0, C_{0} I_{m} \otimes I_{k}\right)$. The spherical normal distribution $N_{m \times k}\left(0, C_{0} I_{m} \otimes I_{k}\right)$ is 
clearly invariant under permutation of the rows of the matrix. Hence, the induced prior distribution of $\beta \beta^{\prime}$ and of the covariance matrix $\Sigma$ from 1.2 is invariant under simultaneous permutation of rows and columns.

Working with the prior just described comes at the cost of losing the identifiability of $\beta$. However, this can be overcome as follows. Assuming that $m \geq k$, any $m \times k$ matrix $\beta$ with linearly independent columns can be uniquely decomposed as $\beta=L Q$, where $L$ is an $m \times k$ lower triangular matrix with positive diagonal, and $Q$ is a $k \times k$ orthogonal matrix. We may then use the implied distribution of the lower triangular matrix $L$ as a prior on the loading matrix. The following theorem about the joint distribution of $L$ and $Q$ is adapted from Theorem 2.1.13 in Muirhead (1982).

Theorem 2.1. Let $\beta=L Q$ be the $L Q$ decomposition of the $m \times k$ random matrix $\beta \sim N_{m \times k}\left(0, C_{0} I_{m} \otimes I_{k}\right)$, where $m \geq k$. Then the lower triangular matrix $L$ and the orthogonal matrix $Q$ are independent, the distribution of $Q$ is the normalized Haar measure, and the distribution of $L=\left(L_{i j}\right)$ has joint density proportional to

$$
\prod_{i=1}^{m} \prod_{j=1}^{\min \{i, k\}} \exp \left\{-\frac{1}{2 C_{0}} L_{i j}^{2}\right\} \times \prod_{i=1}^{k} L_{i i}^{k-i} \mathbf{1}_{\left\{L_{i i}>0\right\}}
$$

with respect to the Lebesgue measure on the space of $m \times k$ lower triangular matrices.

The joint distribution for the entries of $L=\left(L_{i j}\right)$ given by 2.1 has the entries $L_{i j}, i \geq j$, independent with $L_{i j} \sim N\left(0, C_{0}\right)$ if $i>j$ and $L_{i i}$ following the distribution with density proportional to

$$
x^{k-i} \exp \left\{-\frac{1}{2 C_{0}} x^{2}\right\}, \quad x>0 .
$$

Note that $L_{k k} \sim T N\left(0, C_{0}\right)$. The joint distribution for a lower triangular matrix in (2.1) thus differs from that given by 1.3 only in the coordinates $L_{i i}$ for $1 \leq i \leq$ $k-1$, which are no longer truncated normal.

Assume as in 1.4 that $\Omega$ and $\beta$ are independent a priori. Then since $Q$ is independent of $L$, and

$$
\Sigma=\Omega+\beta \beta^{\prime}=\Omega+L Q Q^{\prime} L^{\prime}=\Omega+L L^{\prime}
$$

does not depend on $Q$, the tuple $(y, \Omega, L, \Sigma)$ is independent of $Q$. Hence, $(\Omega, L, \Sigma)$ is also independent of $Q$ a posteriori (i.e., conditional on $y$ ). Our proposal is now simply to keep with the standard identifiability constraint that has the loading matrix $\beta$ lower triangular with nonnegative diagonal entries but to use the distribution given by 2.1) instead of (1.3) for this lower triangular loading matrix. Concerning the remaining parts of the prior specification, we continue to assume independence of $\beta$ and $\Omega$, and we stick with the choice from $(1.4)$ for the prior on the uniquenesses. This proposed prior has then the property that the distributions of $\beta \beta^{\prime}$ and the covariance matrix $\Sigma$ are invariant under reordering of the variables (i.e., matrix rows and columns), both a priori and a posteriori.

Remark 1. Our presentation treats the prior variance $C_{0}$ as a fixed hyperparameter. Instead, one may wish to introduce a hyper-prior for $C_{0}$. The above approach of integrating out the orthogonal matrix $Q$ remains valid in this extension because the distribution of the orthogonal matrix $Q$ in Theorem 2.1 does not depend on $C_{0}$. So, in a Gibbs sampler as discussed in Section 3 , an update of $C_{0}$ can be based on the conditional distribution of $C_{0}$ given $(\Omega, L, y)$ as well as latent factors $f$. 


\section{GiBBS SAMPLER}

Consider an actual inferential setting in which we observe a sample $y_{1}, \ldots, y_{n}$ that comprises $n$ independent random vectors drawn from a distribution in the $k$ factor model. Let $Y$ be the $n \times m$ matrix with the vectors $y_{1}, \ldots, y_{n}$ as rows. Let $F$ be an associated $n \times k$ matrix whose rows $f_{1}, \ldots, f_{n}$ are independent vectors of latent factors. The factor analysis model dictates that

$$
Y=F \beta^{\prime}+E,
$$

where $E=\left(\varepsilon_{1}, \ldots, \varepsilon_{n}\right)^{\prime}$ is an $n \times m$ matrix of stochastic errors. The pairs $\left(f_{t}, \varepsilon_{t}\right)$ for $1 \leq t \leq n$ are independent, and in each pair $f_{t} \sim N_{k}\left(0, I_{k}\right)$ and $\varepsilon_{t} \sim N_{m}(0, \Omega)$ are independent as well. The unknown parameters are comprised in the matrices $\Omega=\operatorname{diag}\left(\omega_{1}^{2}, \ldots, \omega_{m}^{2}\right)$ and $\beta=\left(\beta_{i j}\right) \in \mathbb{R}^{m \times k}$, where the latter is restricted to be lower triangular with nonnegative diagonal.

We now adopt the prior distribution on $\beta$ and $\Omega$ given by (2.1) and (1.4), and derive the full conditionals needed for a Gibbs sampler that generates draws from the posterior distribution of $(\beta, \Omega)$. As in Lopes and West (2004), we write

$$
\beta_{i}= \begin{cases}\left(\beta_{i 1}, \ldots, \beta_{i i}\right)^{\prime} & \text { if } i \leq k, \\ \left(\beta_{i 1}, \ldots, \beta_{i k}\right)^{\prime} & \text { if } i>k,\end{cases}
$$

and explicitly involve the latent factors in $F$. Let $F_{i}$ be the $n \times i$ matrix made up of the first $i$ columns of $F$, and write $Y_{i}$ for the $i$-th column of $Y$ (in contrast to $y_{t}$, which is the $t$-th row of $Y$ ). The full conditionals for $F, \Omega$ and $\beta$ are determined as follows. First, the rows $f_{t}$ of $F$ are conditionally independent given $(\beta, \Omega, Y)$ with

$$
\left(f_{t} \mid \beta, \Omega, Y\right) \sim N_{k}\left(\left(I_{k}+\beta^{\prime} \Omega^{-1} \beta\right)^{-1} \beta^{\prime} \Omega^{-1} y_{t},\left(I_{k}+\beta^{\prime} \Omega^{-1} \beta\right)^{-1}\right)
$$

for $t=1, \ldots, n$. Second, the uniquenesses $\omega_{1}^{2}, \ldots, \omega_{m}^{2}$ are conditionally independent given $(\beta, F, Y)$ with

$$
\left(\omega_{i}^{2} \mid \beta, F, Y\right) \sim I G\left(\frac{1}{2}(\nu+T), \frac{1}{2}\left(\nu s^{2}+d_{i}\right)\right),
$$

where

$$
d_{i}=\left(Y_{i}-F_{i} \beta_{i}^{\prime}\right)^{\prime}\left(Y_{i}-F_{i} \beta_{i}^{\prime}\right) .
$$

Third, the rows of $\beta$ are conditionally independent given $(\Omega, F, Y)$. For $i=1, \ldots, k$, the conditional density of the vector $\beta_{i}$ is proportional to

$$
\beta_{i i}^{k-i} \frac{1}{\operatorname{det}\left(C_{i}\right)} \exp \left\{-\frac{1}{2}\left(\beta_{i}-m_{i}\right)^{\prime} C_{i}^{-1}\left(\beta_{i}-m_{i}\right)\right\} \mathbf{1}_{\left\{\beta_{i i}>0\right\}}
$$

where

$$
C_{i}=\left(\frac{1}{C_{0}} I_{i}+\frac{1}{\omega_{i}^{2}} F_{i}^{\prime} F_{i}\right)^{-1} \text { and } m_{i}=\frac{1}{\omega_{i}^{2}} C_{i} F_{i}^{\prime} Y_{i} .
$$

For $i=k+1, \ldots, m$, the conditional distribution is

$$
\left(\beta_{i} \mid \Omega, F, Y\right) \sim N_{k}\left(m_{i}, C_{i}\right)
$$

with

$$
C_{i}=\left(\frac{1}{C_{0}} I_{k}+\frac{1}{\omega_{i}^{2}} F^{\prime} F\right)^{-1} \text { and } m_{i}=\frac{1}{\omega_{i}^{2}} C_{i} F^{\prime} Y_{i} .
$$

The only full conditional that differs from those given in Lopes and West (2004) is the one for $\beta_{i}$ with $i \leq k$ from (3.4). To draw from this distribution, we first sample from $\left(\beta_{i i} \mid \Omega, F, Y\right)$ and then from $\left(\beta_{i 1}, \ldots, \beta_{i i-1} \mid \beta_{i i}, \Omega, F, Y\right)$. The latter 
distribution is a multivariate normal distribution. The only new challenge is thus the sampling from $\left(\beta_{i i} \mid \Omega, F, Y\right)$, which has density proportional to

$$
\beta_{i i}^{k-i} e^{-\frac{\left(\beta_{i i}-a\right)^{2}}{2 b^{2}}} \mathbf{1}_{\left\{\beta_{i i}>0\right\}}
$$

for constants $a \in \mathbb{R}$ and $b>0$ determined by $(\Omega, F, Y)$. After scaling $\beta_{i i}$ by $b$, the problem reduces to generating draws from distributions with density in the class

$$
f(x \mid \alpha, \gamma)=\frac{1}{Z(\alpha, \gamma)} x^{\alpha-1} e^{-(x-\gamma)^{2}}, \quad x>0,
$$

where $\alpha>0$ and $\gamma \in \mathbb{R}$ are two parameters, and $Z(\alpha, \gamma)$ is the normalizing constant. In the present context, integer values of $\alpha$ are of interest. The densities in (3.6) are log-concave, and we will use adaptive rejection sampling (Gilks and Wild, 1992) as implemented in the $\mathrm{R}$ package ars to draw samples from them. This said, it would be interesting to develop special samplers for this class of distributions.

As pointed out by a reviewer, a slice sampler could be set up by introducing an auxiliary variable $u$ with joint density

$$
f(x, u \mid \alpha, \gamma) \propto x^{\alpha-1} \mathbf{1}_{\left\{0<u<e^{\left.-(x-\gamma)^{2}\right\}}\right.}, \quad x>0 .
$$

The sampler would iteratively generate draws from the conditional distribution of $x$ given $u$, which be of truncated Gamma type (Philippe, 1997), and from the conditional distribution of $u$ given $x$, which is uniform.

However, it should also be possible to design efficient rejection samplers. A detailed discussion is beyond the scope of this paper but we note that when $\gamma=0$ then the density from (3.6) can be transformed into a Gamma density by taking squares. Moreover, the distribution defined by (3.6) can be approximated by a normal distribution when $\gamma \rightarrow \infty$ and by a Gamma distribution when $\gamma \rightarrow-\infty$.

\section{Numerical experiments}

We illustrate the use of the two different priors, obtained from (1.3) and (2.1), respectively, on a simulated dataset $Y$ that involves $m=15$ variables and is of size $n=30$. The data are drawn from the $k=3$ factor distribution given by the following loading matrix and uniquenesses that we generated randomly:

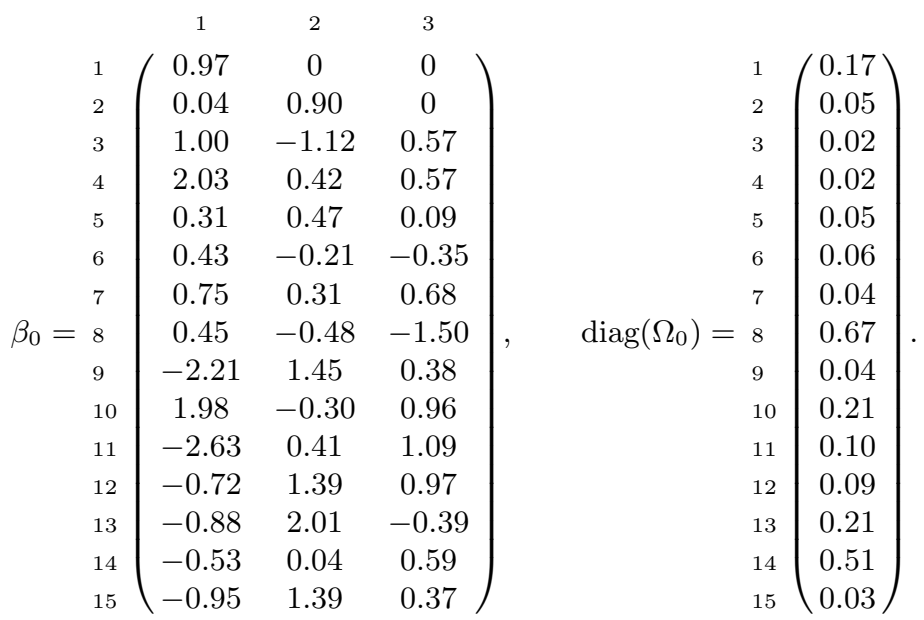

We create a second data matrix $Y^{\pi}$ by permuting the columns of $Y$ based on the permutation $\pi$ from Table 1. The $i$-th column of $Y$ becomes the $\pi(i)$-th column 
TABLE 1. The permutation $\pi$ used to reorder simulated data.

\begin{tabular}{c|rrrrrrrrrrrrrrr}
\hline \hline$i$ & 1 & 2 & 3 & 4 & 5 & 6 & 7 & 8 & 9 & 10 & 11 & 12 & 13 & 14 & 15 \\
\hline$\pi(i)$ & 10 & 14 & 13 & 15 & 12 & 6 & 7 & 2 & 11 & 9 & 8 & 3 & 5 & 1 & 4 \\
\hline
\end{tabular}

of $Y^{\pi}$. We set the hyperparameters as in Lopes and West (2004), namely, $C_{0}=1$, $\nu=2.2$ and $s=\sqrt{0.1 / 2.2}$. Via Gibbs sampling as described in Section 3 , we draw from the posterior distributions for the covariance matrix $\Sigma=\left(\sigma_{i j}\right)=\Omega+\beta \beta^{\prime}$ for each data set, focusing on the models with $k=3$ and $k=6$ factors. The Gibbs samplers are initialized at the respective maximum likelihood estimates for $(\beta, \Omega)$. After a burnin of 10,000 iterations, we ran each sampler for 300,000 iterations.

Figures 4.1 and 4.2 show kernel density estimates of the posterior densities of selected variances. More precisely, we compare the densities of $\left(\sigma_{i i} \mid Y\right)$ and $\left(\sigma_{\pi(i), \pi(i)} \mid Y^{\pi}\right)$ for $i=1,8,14$. Under our proposed prior from (2.1), the two posterior densities are the same. Indeed, the plots in the right hand columns of Figures 4.1 and 4.2 show only minor discrepancies due to Monte Carlo error. The 'standard prior' from (1.3), however, results in visible differences that are more pronounced for $k=6$, which is not surprising as larger differences are possible among the degrees of freedom of the chi-square prior for $\left(\beta \beta^{\prime}\right)_{i i} / C_{0}$; recall (1.5). Note that the observed shifts in the posterior distributions under the 'standard prior' are explained by the different chi-square degrees of freedom.

We also compare the posterior means of the covariance matrices for $Y$ and $Y^{\pi}$ under both prior schemes. Let $\Sigma^{\pi}$ be the matrix whose rows and columns are repermuted so that $\left(\Sigma^{\pi}\right)_{i j}=\sigma_{\pi(i), \pi(j)}$. In other words, both $\Sigma$ and $\Sigma^{\pi}$ have their rows and columns ordered in the same way as the variables in the dataset $Y$ are ordered. Figure 4 shows heat maps of the matrix $\mathbb{E}\left(\Sigma^{\pi} \mid Y^{\pi}\right)-\mathbb{E}(\Sigma \mid Y)$ under the models with $k=3$ and 6 , respectively. As expected, the entries of the matrix $\mathbb{E}\left(\Sigma^{\pi} \mid Y^{\pi}\right)-\mathbb{E}(\Sigma \mid Y)$ have larger magnitude under the 'standard prior'. For both $k=3$ and $k=6$ the largest discrepancy occurs for $\sigma_{14,14}$, which can be explained by the fact that the permutation $\pi$ makes variable 14 the first variable.

\section{Conclusion}

In Bayesian inference in exploratory factor analysis, priors are often specified via a lower triangular loading matrix $\beta$ whose entries are assumed to be independent normal or truncated normal. We propose a modification of this approach, replacing the truncated normal priors by other distributions such that the induced prior on the covariance matrix $\Sigma$ in 1.2 is invariant under reordering of the considered variables. Specifically, the prior distribution of $\beta \beta^{\prime}$ is equal to that obtained when letting $\beta$ be a $m \times k$ matrix filled with $m k$ i.i.d. centered normal random variables.

Invariance of the prior, and thus also the posterior for the covariance $\Sigma$ ensures that inference on covariance parameters, i.e., functions of $\Sigma$, does not depend on how variables are ordered in the dataset. We emphasize that while we have used lower triangular loading matrices as a device for computation, other definitions of loading matrices could be adopted to define the target of inference; for sampling-based approximation of their posterior distributions one simply computes these covariance parameters from the output of the Gibbs sampler we described. Furthermore, using the proposed priors in Bayesian model selection (Lopes and West, 2004) yields inferences on the number of factors $k$ that do not depend on the variable ordering. 

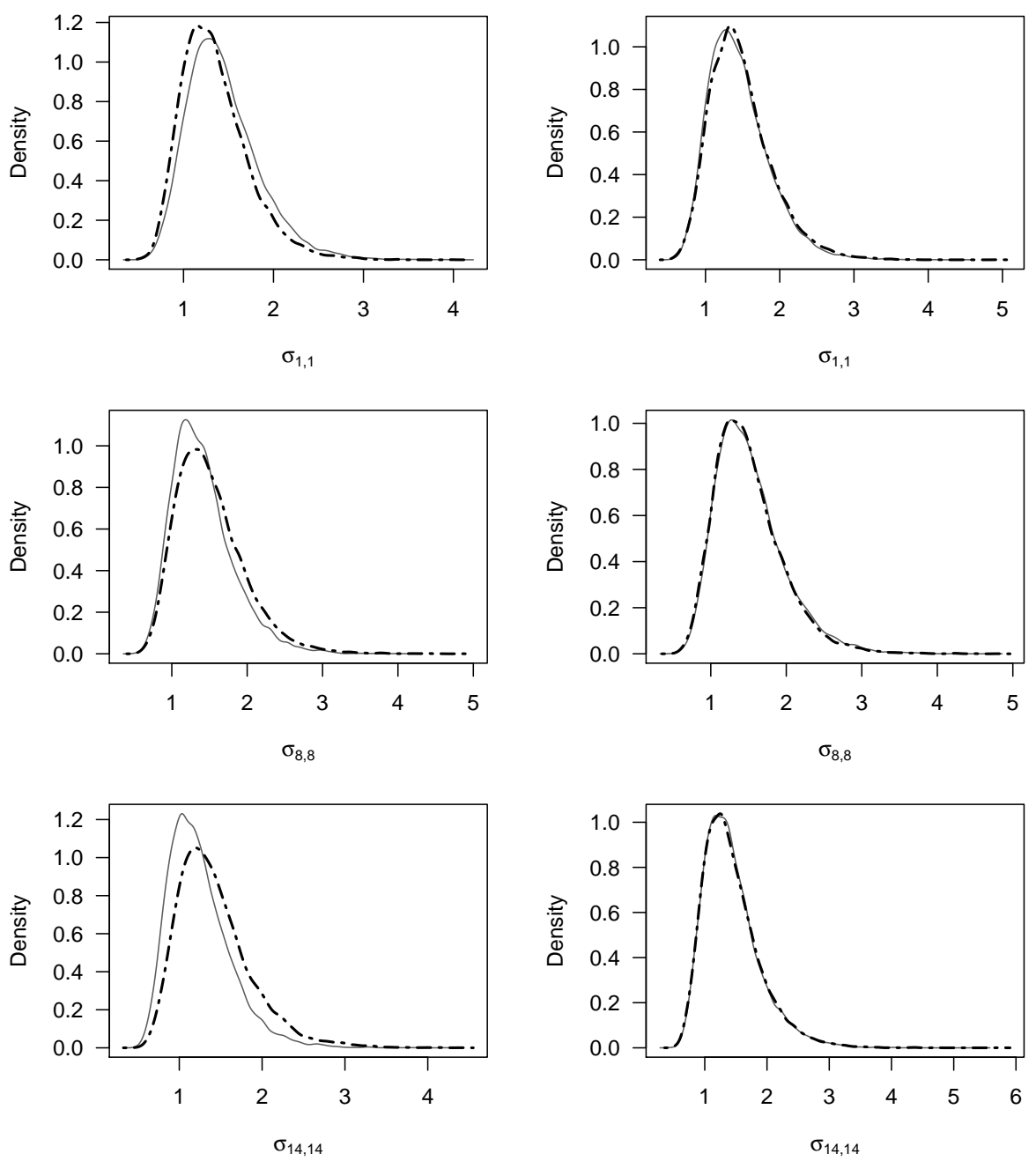

Figure 4.1. Posterior densities of $\left(\sigma_{i i} \mid Y\right)$, in black, and of $\left(\sigma_{\pi(i), \pi(i)} \mid Y^{\pi}\right)$, in grey, in factor analysis with $k=3$ factors, for $i=1,8,14$. The left column concerns the prior from 1.3 , and the right column is based on the prior proposed in 2.1 .

The proposed prior is intended as a possible default when there is no reason to impose dependence among loadings or to treat the loadings of different variables differently. Concerning possible departures from our default scenario, we remark that the software of Martin et al. (2011) also allows one to impose patterns of zeros in the loading matrix $\beta$. As mentioned earlier, the latter situation is sometimes termed confirmatory factor analysis. The identifiability issues we addressed do not arise in the same way in the confirmatory setting as orthogonal transformations will generally not preserve prescribed zeros in the loading matrix.

Sampling from the posterior distribution resulting from the prior we proposed is largely the same as for the 'standard prior' that has been used by several authors 

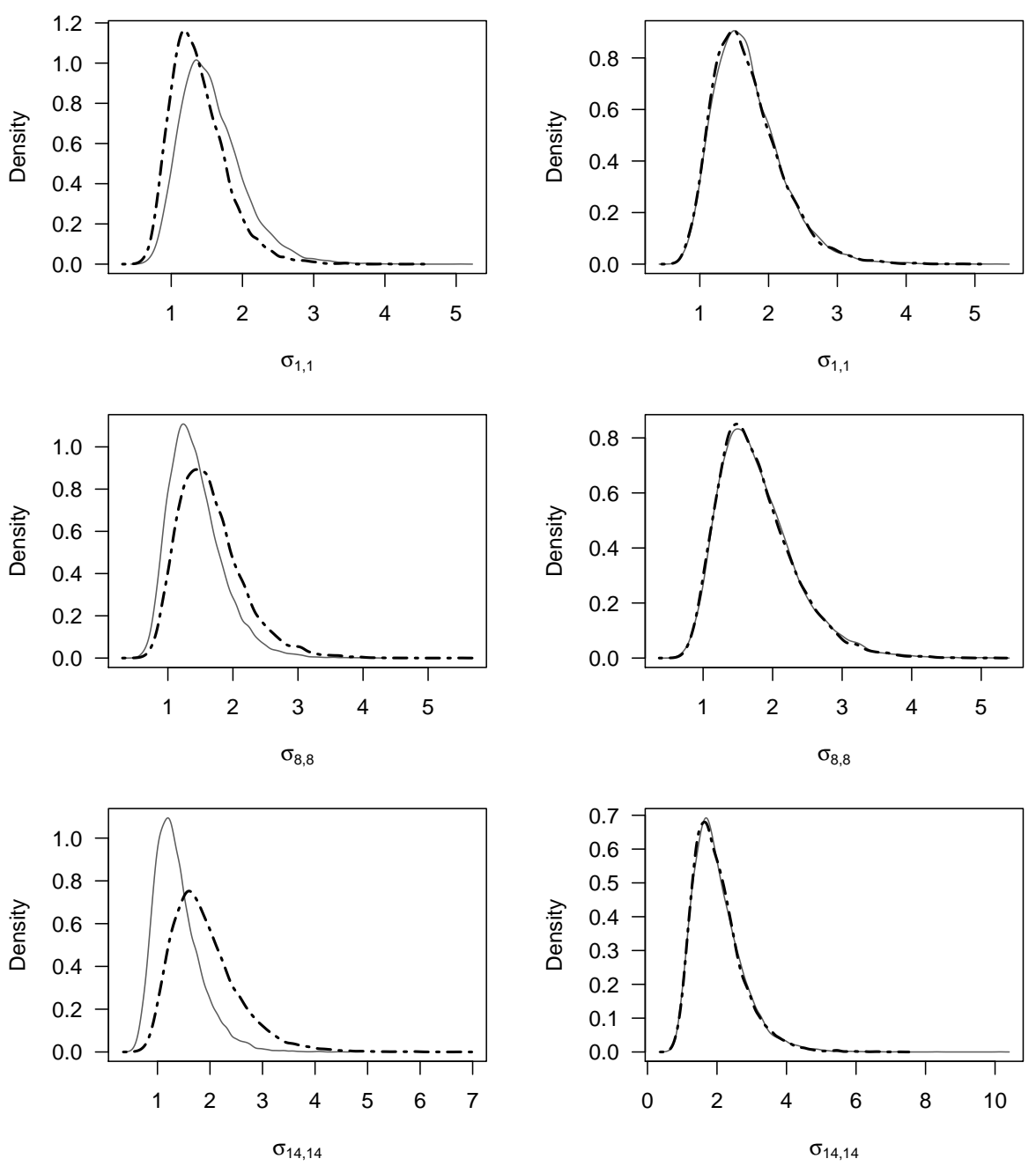

Figure 4.2. Posterior densities of $\left(\sigma_{i i} \mid Y\right)$, in black, and of $\left(\sigma_{\pi(i), \pi(i)} \mid Y^{\pi}\right)$, in grey, in factor analysis with $k=6$ factors, for $i=1,8,14$. The left column concerns the prior from 1.3 , and the right column is based on the prior proposed in 2.1 .

including Geweke and Zhou (1996) and Lopes and West (2004). The key difference is the need to sample from distributions in the class specified by $(3.6)$. These distributions also appear in the realm of multivariate $t$-distributions (Finegold and Drton, 2011, 2014), although a square-root transformation is necessary to match the setup there. As mentioned at the end of Section 3, it seems worthwhile to develop an efficient sampler targeting precisely this family of distributions.

\section{ACKNowledgments}

This work was supported by the U.S. National Science Foundation (DMS-1305154) and by the University of Washington Royalty Research Fund. 
a)
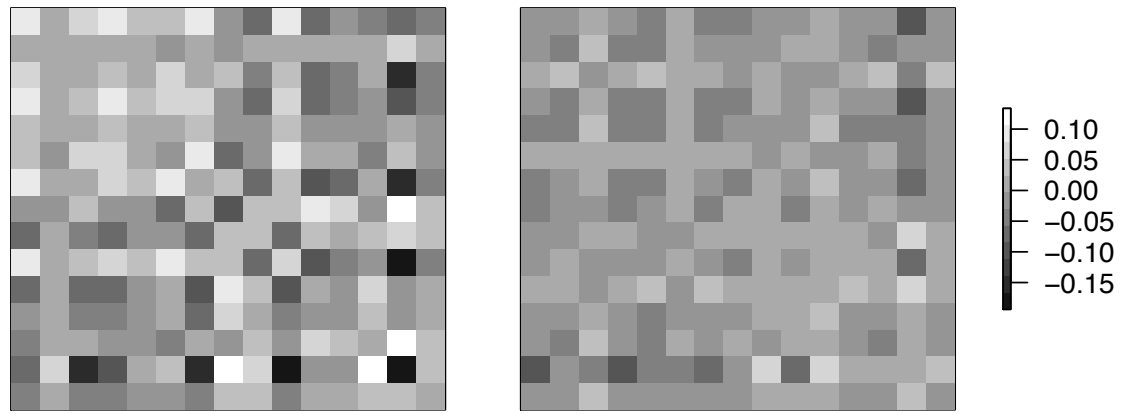

b)
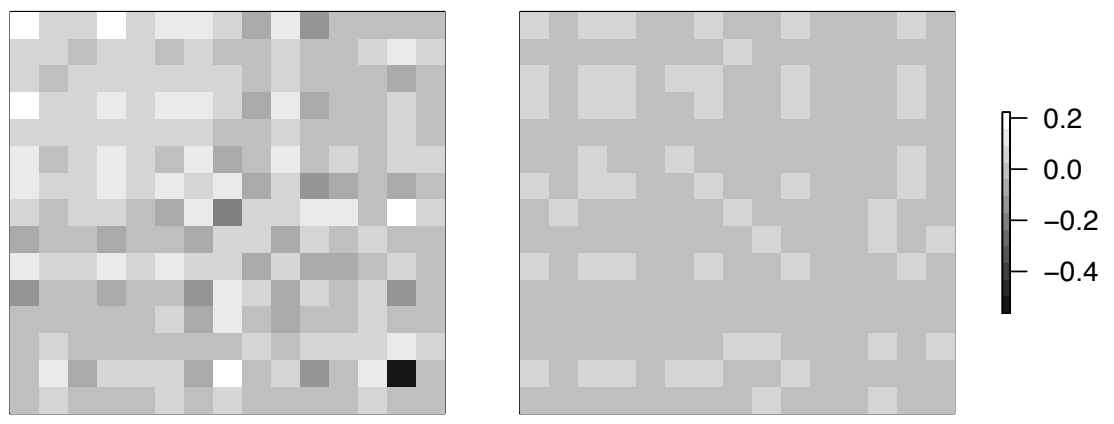

Figure 4.3. Heat maps of $\mathbb{E}\left(\Sigma^{\pi} \mid Y^{\pi}\right)-\mathbb{E}(\Sigma \mid Y)$ in the models with a) $k=3$ and b) $k=6$ factors. On the left: Results for the 'standard prior' from (1.3). On the right: results for the prior from 2.1).

\section{REFERENCES}

Aguilar, O. and West, M. (2000). "Bayesian dynamic factor models and variance matrix discounting for portfolio allocation." J. Bus. Econom. Statist., 18(3): 338-357.

Anderson, T. W. and Rubin, H. (1956). "Statistical inference in factor analysis." In Proceedings of the Third Berkeley Symposium on Mathematical Statistics and Probability, 1954-1955, vol. V, 111-150. University of California Press, Berkeley and Los Angeles.

Ando, T. (2009). "Bayesian factor analysis with fat-tailed factors and its exact marginal likelihood." J. Multivariate Anal., 100(8): 1717-1726.

Ansari, A., Jedidi, K., and Dube, L. (2002). "Heterogeneous factor analysis models: a Bayesian approach." Psychometrika, 67(1): 49-78.

Bartholomew, D., Knott, M., and Moustaki, I. (2011). Latent variable models and factor analysis. Wiley Series in Probability and Statistics. John Wiley \& Sons, Ltd., Chichester, third edition. A unified approach.

Bhattacharya, A. and Dunson, D. B. (2011). "Sparse Bayesian infinite factor models." Biometrika, 98(2): 291-306.

Congdon, P. (2001). Bayesian statistical modelling. Wiley Series in Probability and Statistics. John Wiley \& Sons, Ltd., Chichester.

Conti, G., Frühwirth-Schnatter, S., Heckman, J. J., and Piatek, R. (2014). "Bayesian exploratory factor analysis." J. Econometrics, 183(1): 31-57.

Drton, M., Sturmfels, B., and Sullivant, S. (2007). "Algebraic factor analysis: tetrads, pentads and beyond." Probab. Theory Related Fields, 138(3-4): 463-493.

Finegold, M. and Drton, M. (2011). "Robust graphical modeling with classical and alternative $t$-distributions." Annals of Applied Statistics, 5(2A): 1057-1080. 
- (2014). "Robust Bayesian graphical modeling using Dirichlet $t$-distributions." Bayesian Analysis, 9(3): 521-550.

Geweke, J. and Zhou, G. (1996). "Measuring the pricing error of the arbitrage pricing theory." The Review of Financial Studies, 9(2): 557-587.

Ghosh, J. and Dunson, D. B. (2009). "Default prior distributions and efficient posterior computation in Bayesian factor analysis." J. Comput. Graph. Statist., 18(2): 306-320.

Gilks, W. R. and Wild, P. (1992). "Adaptive rejection sampling for Gibbs sampling." Applied Statistics, 41(2): 337-348.

Lopes, H. F. and West, M. (2004). "Bayesian model assessment in factor analysis." Statist. Sinica, 14(1): 41-67.

Martin, A. D., Quinn, K. M., and Park, J. H. (2011). "MCMCpack: Markov Chain Monte Carlo in R." Journal of Statistical Software, 42(9): 22.

Muirhead, R. J. (1982). Aspects of multivariate statistical theory. John Wiley \& Sons, Inc., New York. Wiley Series in Probability and Mathematical Statistics.

Mulaik, S. A. (2010). Foundations of factor analysis. Statistics in the Social and Behavioral Sciences Series. CRC Press, Boca Raton, FL, second edition.

Nakajima, J. and West, M. (2013). "Bayesian analysis of latent threshold dynamic models." J. Bus. Econom. Statist., 31(2): 151-164.

Paisley, J. and Carin, L. (2009). "Nonparametric factor analysis with beta process priors." In Proceedings of the International Conference on Machine Learning, 777-784.

Philippe, A. (1997). "Simulation of right and left truncated gamma distributions by mixtures." Statistics and Computing, 7(3): 173-181.

Zhou, X., Nakajima, J., and West, M. (2014). "Bayesian forecasting and portfolio decisions using dynamic dependent sparse factor models." Int. J. Forecasting, 30: 963-980.

Department of Statistics, University of Washington, Seattle, WA, U.S.A.

E-mail address: dmhleung@uw.edu

Department of Statistics, University of Washington, Seattle, WA, U.S.A.

E-mail address: md5@uw.edu 